\title{
No-go theorems on localization of gravity around higher codimensional branes in noncompact extra dimensions
}

\section{Shing Yan Li}

Center for Theoretical Physics, Department of Physics, Massachusetts Institute of Technology, 77 Massachusetts Ave, Cambridge, MA 02139, U.S.A.

Perimeter Institute for Theoretical Physics,

31 Caroline St. N, Waterloo, ON N2L 2Y5, Canada

Department of Physics, Hong Kong University of Science and Technology,

Clear Water Bay, Kowloon, Hong Kong

E-mail: sykobeli@mit.edu

ABSTRACT: We study the brane world scenario of a single brane (or a single stack of branes) with codimension higher than one. When the extra dimensions are not small, localization of gravity around the brane is needed in order to reproduce the observable four-dimensional gravity. We focus on the case of noncompact extra dimensions, where the possibility of localized gravity becomes non-trivial. We show that in large class of gravity models, localization of massless gravity is not possible for codimension-2 branes with at least one noncompact extra dimension. With additional mild assumptions on field backgrounds, we also show that it is not possible for higher codimensional branes with two or more noncompact extra dimensions.

KEYworDs: Large Extra Dimensions, p-branes, Supergravity Models

ArXiv EPrint: 2012.06409 


\section{Contents}

1 Introduction 1

2 Codimension-2 branes 3

2.1 General setup 3

2.2 Exactly solvable models 6

2.3 Necessary conditions for existence of massless localized graviton 8

$\begin{array}{ll}2.4 \text { No-go theorems } & 9\end{array}$

3 Branes with higher codimensions $\quad 11$

3.1 Klebanov-Strassler throat 11

$\begin{array}{lll}3.2 & \text { General setup } & 13\end{array}$

$\begin{array}{lll}3.3 & \text { No-go theorem } & 15\end{array}$

$\begin{array}{lll}4 & \text { Conclusion } & 17\end{array}$

\section{Introduction}

Extra dimensions are well-motivated by, for example, string theory and have rich and novel impacts on phenomenology, such as providing potential solutions to hierarchy problems in particle physics and cosmology. Some well-known examples are the cosmological constant problem [1-13] and the Higgs hierarchy problem [14-19]. On the other hand, mechanisms are needed to implement a noncompact four-dimensional spacetime into the extra dimensions, in order to be consistent with our observations. So far there are two well-known ways, namely compactification and the brane world scenario.

In the former case, the extra dimensions are "packed" into tiny compact space, such that they are too small to be seen within the energy scale we can achieve. In the latter case, a space-filling 3-brane sits in the extra dimensions called bulk, and our universe is trapped on the brane. While the extra dimensions must be small in compactification, those in the brane world scenario can be large or even infinitely large i.e. noncompact. In this paper, we investigate how noncompact extra dimensions can affect the low-energy physics, in order to judge the validity of these theories.

An essential feature of such theories is localization of gravity, which can be explained as follows. The matter (Standard Model) particles can be naturally trapped onto the brane, such as in the context of D-branes. Therefore these particles can "see" fewer dimensions. However, gravity is dynamics of spacetime, and can freely propagate through the whole spacetime. Therefore to recover the observed Newton's law, which implies four-dimensional gravity, mechanisms of localization of gravity is needed to build the brane world scenario. For simplicity, we always focus on localized massless gravity only, although massive gravity 
is not completely ruled out by phenomenology. As we will see, it is always possible in large but compact extra dimensions, while it is hard in noncompact extra dimensions due to nontrivial boundary conditions. Some aspects of localized gravity with higher codimensions are already studied in literature such as in [20-23], but most of them focused on compact extra dimensions only, and did not have a concrete conclusion on the case of noncompact extra dimensions.

For codimension-1 brane, localization of gravity is easily achieved in the RandallSundrum model [24], where the four-dimensional massless graviton is localized by a warp factor induced by fine-tuning between positive brane tension and negative bulk cosmological constant. It is later generalized to the codimension-1 Karch-Randall model [25]. It is then natural to ask whether it is also possible with higher codimensions. It is non-trivial because they have distinct dynamics. Codimension- 1 sources usually cause a jump of fields at brane position, determined by the Israel junction conditions [26]. However, higher codimensional sources generically cause divergences at brane position. Such behavior is similar to the Coulomb potential in dimension higher than one, which divergences are caused at charge positions. This is why the dynamics of codimension- 1 brane are much more well studied in literature. Techniques to regularize these divergences for codimension-2 branes are developed in [27-29], and it is straightforward to generalize them to even higher codimensions.

In this paper, we show that generically there is no localized gravity around codimension-2 branes with at least one noncompact extra dimension, or higher codimensional branes with at least two noncompact extra dimensions. We consider a general theory with Einstein gravity, form field backgrounds and dilaton, which includes large class of supergravity models. We prove that in generic background, the warp factor far from the brane cannot vanish asymptotically, thus cannot localize or normalize the massless graviton mode function. We also derive the brane-bulk boundary conditions which limit the form of the mode function, implying that localized gravity can only be achieved by warp factors. Hence localization of gravity is not possible. To achieve so, we use techniques from analysis to study the general features of the field equations without solving them. As a corollary, we also show that when a codimension-1 localized gravity model actually has one compactified hidden dimension, within our model the brane must also wrap that dimension. Note that we only consider a single brane (or a single stack of branes). This simplifies the calculation much, and excludes more complicated setups of higher codimensions such as brane intersections.

This paper is organized as follows. In section 2, we use codimension- 2 branes as example to study the dynamics among branes, noncompact extra dimensions and massless graviton modes. We review a general setup of such models, which can be easily generalized into higher codimensions. We then examine the boundary conditions to determine the consistent way to localize gravity, which is by warp factor. After that, we prove the nogo theorem for codimension-2 branes. We prove it separately for the cases of one and two noncompact extra dimensions. This includes the case where a Randall-Sundrum-like solution with a codimension- 2 brane seems to trivially appear. We show that such solution does not exist. 
In section 3, we study localized gravity around higher codimensional branes in general. To build motivation to study such no-go theorems from some realistic models, we first use a (infinitely long) Klebanov-Strassler throat [30, 31], which is a well-known string theory solution, as an example. We then generalize the setup of the system and prove the no-go theorem for higher codimensions. To achieve so, additional assumptions on the background must be made. Namely, the background is non-oscillating, and under presence of form fields with nonvanishing couplings or brane curvature.

In section 4, we conclude and add some remarks to our results.

\section{Codimension-2 branes}

In this section, we use codimension-2 branes as example to demonstrate general features of the dynamics of brane-bulk system and gravitons. From these we prove the no-go theorems for codimension-2 branes.

\subsection{General setup}

Brane-bulk system. We first establish the brane-bulk system, which is most reviewed in [29]. For the bulk, we consider a simple model of scalar-Einstein-Maxwell system with single fields. Similar but more complicated arguments should hold for the cases of multiple fields, so the model below is general enough to describe the bosonic parts of large class of supergravity models. To implement the $4 \mathrm{D}$ universe, there is a maximally symmetric 3 -brane sitting in the bulk. The action (in Einstein frame) is given by ${ }^{1}$

$$
\begin{aligned}
S & =S_{\text {bulk }}+S_{\text {brane }} \\
S_{\text {bulk }} & =-\int d^{4} x d^{2} y \sqrt{-g}\left(\frac{1}{2 \kappa^{2}} R+\frac{1}{2} \partial^{M} \phi \partial_{M} \phi+\frac{1}{4} c(\phi) F_{M N} F^{M N}+V(\phi)\right)+S_{\mathrm{GH}} \\
S_{\text {brane }} & =-\int_{y_{b}} d^{4} x \sqrt{-\bar{g}} T_{b},
\end{aligned}
$$

where $g$ is the $6 \mathrm{D}$ background metric, $\bar{g}$ is the unwarped $4 \mathrm{D}$ background metric, $R$ is the $6 \mathrm{D}$ Ricci scalar, $\phi$ is a scalar field, $F=d A$ is the Maxwell field strength, $c>0$ is the field-dependent inversed coupling of the Maxwell field, $V$ is the bulk potential including a bulk cosmological constant and the scalar field potential, $T_{b}$ is the warped brane potential and $y_{b}$ is the position of the brane. $S_{\mathrm{GH}}$ is the Gibbons-Hawking action at the brane-bulk boundary, added to restore the usual Einstein equations. We first consider two noncompact extra dimensions, since we will generalize such setup to higher codimensions. For a single brane, the bulk geometry is rotationally invariant. We also include warped geometries. Therefore the metric $g$ can be written as

$$
d s^{2}=g_{M N} d x^{M} d x^{N}=e^{2 A(r)} \bar{g}_{\mu \nu} d x^{\mu} d x^{\nu}+d r^{2}+e^{2 B(r)} d \theta^{2},
$$

\footnotetext{
${ }^{1}$ Our metric is mostly plus, with Weinberg's curvature conventions [32], which differ from those of MTW [33] only by an overall sign in the definition of the Riemann tensor.
} 
where $A$ and $B$ are the warp factors which only depend on $r$ and $\bar{g}$ is the maximally symmetric metric. In terms of static coordinates,

$$
\bar{g}_{\mu \nu} d x^{\mu} d x^{\nu}=-\left(1-k \rho^{2}\right) d t^{2}+\frac{d \rho^{2}}{1-k \rho^{2}}+\rho^{2}\left(d \varphi_{1}^{2}+\sin ^{2} \varphi_{1} d \varphi_{2}^{2}\right) .
$$

The parameter $k=\{-1,0,1\}$ corresponds to unit-radius AdS, flat and dS spacetime respectively. To obtain two noncompact extra dimensions, $r$ should go from zero to infinity, and the warp factor $B$ should satisfy $e^{B} \rightarrow+\infty$ as $r \rightarrow \infty$. It is then natural to assume that $B$ is increasing at large $r . B$ should also satisfy $\lim _{r \rightarrow 0} e^{B}=0$, in order to put the codimension-2 brane at $r=0$. From now on we use capital English letter to denote all coordinates, and Greek letters to denote $4 \mathrm{D}$ coordinates. Now we can choose a gauge such that the only non-vanishing components of $F$ are

$$
F_{r \theta}=-F_{\theta r}=A_{\theta}^{\prime}(r) .
$$

By symmetry $A_{\theta}$ and $\phi$ also depend on $r$ only. Also from symmetry $T_{b}$ should be function of $\phi$ and $A_{\theta}$ only. Therefore we perform a derivative expansion to get

$$
T_{b}=\tau_{b}(\phi)-\frac{1}{2} \Phi_{b}(\phi) \epsilon^{m n} F_{m n}+\ldots,
$$

where $\tau_{b}$ is the warped brane tension and $\Phi_{b}$ is the warped localized magnetic flux on the brane [34]. The labels $m, n$ are for $r, \theta$ and $\epsilon_{m n}$ is the corresponding Levi-Civita tensor.

Now for $r>0$, the bulk field equations are

$$
\begin{aligned}
\phi^{\prime \prime}+\left(B^{\prime}+4 A^{\prime}\right) \phi^{\prime} & =\frac{\partial V}{\partial \phi}+\frac{1}{4} \frac{\partial c}{\partial \phi} F_{M N} F^{M N}, \\
R_{M N}-\frac{1}{2} R g_{M N} & =-c \kappa^{2}\left(F_{M P} F_{N}^{P}-\frac{1}{4} g_{M N} F_{P Q} F^{P Q}\right)-\kappa^{2}\left(\partial_{M} \phi \partial_{N} \phi-g_{M N}\left(\frac{1}{2} \partial^{P} \phi \partial_{P} \phi+V\right)\right), \\
\nabla_{M}\left(c F^{M N}\right) & =0 .
\end{aligned}
$$

Using the above ansatz, we get

$$
\left(c e^{-B+4 A} A_{\theta}^{\prime}\right)^{\prime}=0 \Rightarrow A_{\theta}^{\prime}=\frac{F_{0}}{c} e^{B-4 A},
$$

where $F_{0}$ is an integration constant and "prime" is partial derivative with respect to $r$. We further denote $f=\frac{\kappa^{2} F_{0}^{2}}{2 c}$. It is then straightforward to compute the components of the Einstein equation:

$$
\begin{aligned}
6 A^{\prime 2}+3 A^{\prime} B^{\prime}+B^{\prime 2}+3 A^{\prime \prime}+B^{\prime \prime}+f e^{-8 A}-3 k e^{-2 A}+\kappa^{2}\left(\frac{1}{2} \phi^{2}+V\right) & =0, \quad(\mu \nu) \\
6 A^{\prime 2}+4 A^{\prime} B^{\prime}-f e^{-8 A}-6 k e^{-2 A}+\kappa^{2}\left(-\frac{1}{2} \phi^{2}+V\right) & =0, \quad(r r) \\
10 A^{\prime 2}+4 A^{\prime \prime}-f e^{-8 A}-6 k e^{-2 A}+\kappa^{2}\left(\frac{1}{2} \phi^{2}+V\right) & =0
\end{aligned}
$$


To understand the back-reaction by the brane to the bulk, we also need the brane-bulk matching conditions [27-29], which tells

$$
\begin{aligned}
\lim _{r \rightarrow 0} \oint_{y_{b}} d \theta \sqrt{-g} \phi^{\prime} & =-\frac{\delta S_{\text {brane }}}{\delta \phi}, \\
\lim _{r \rightarrow 0} \oint_{y_{b}} d \theta \sqrt{-g} F^{r M} & =-\frac{\delta S_{\text {brane }}}{\delta A_{M}}, \\
\lim _{r \rightarrow 0} \oint_{y_{b}} d \theta \frac{1}{2 \kappa^{2}} \sqrt{-g}\left(K^{i j}-K g^{i j}\right)-(\text { flat }) & =-\frac{\delta S_{\text {brane }}}{\delta g_{i j}} .
\end{aligned}
$$

Here the extrinsic curvature $K_{i j}$ of fixed-r surface is given by $K_{i j}=\frac{1}{2} \partial_{r} g_{i j}$, with $i, j$ label all coordinates except $r$. The "flat" is the same result substituting $B=\ln r$ and $\lim _{r \rightarrow 0} A^{\prime}=0$ with $A(0)$ unchanged i.e. with a flat metric continuous to metric outside the brane. The integration is along a small circle around the brane. Eq. (2.16) simply relates $\Phi_{b}$ to $F_{0}[12,34]$, and for simplicity we will use $f$ to perform calculations. On the other hand, eq. (2.17) becomes

$$
\begin{aligned}
-\lim _{r \rightarrow 0} \frac{2 \pi}{\kappa^{2}} e^{4 A}\left(e^{B}\left(3 A^{\prime}+B^{\prime}\right)-1\right) & =T_{b}, \quad(\mu \nu) \\
\lim _{r \rightarrow 0} \frac{2 \pi}{\kappa^{2}} e^{B+4 A} A^{\prime}=-\frac{1}{2 \sqrt{-\bar{g}}} \frac{\partial}{\partial g_{\theta \theta}}\left(\sqrt{\bar{g}} T_{b}\right) & =U_{b} . \quad(\theta \theta)
\end{aligned}
$$

From these matching conditions and the field equations, one can derive a constraint [27$29,35]$ in which $U_{b}$ can be fully determined by $T_{b}$ and $\frac{\partial T_{b}}{\partial \phi}$. It reads

$$
\frac{\kappa^{2} U_{b}}{2 \pi}=\frac{1}{3}\left(e^{4 A}-\frac{\kappa^{2} T_{b}}{2 \pi} \pm \sqrt{\left(e^{4 A}-\frac{\kappa^{2} T_{b}}{2 \pi}\right)^{2}-\frac{3}{4}\left(\frac{\kappa^{2}}{2 \pi} \frac{\partial T_{b}}{\partial \phi}\right)^{2}}\right)
$$

where the sign is chosen such that $U_{b} \rightarrow 0$ when $\frac{\partial T_{b}}{\partial \phi} \rightarrow 0$.

Graviton modes. After solving the background metric, we add linear perturbation to solve for $4 \mathrm{D}$ graviton modes $h_{\mu \nu}$. The metric becomes

$$
d s^{2}=e^{2 A(r)}\left(\bar{g}_{\mu \nu}+h_{\mu \nu}\right) d x^{\mu} d x^{\nu}+d r^{2}+e^{2 B(r)} d \theta^{2} .
$$

By symmetry we can separate the variables as $h_{\mu \nu}(x, y)=\bar{h}_{\mu \nu}(x) \psi(y)$, where in the transverse and traceless gauge it satisfies

$$
\bar{h}_{\mu}^{\mu}=\bar{\nabla}^{\mu} \bar{h}_{\mu \nu}=0
$$

where the covariant derivative is with respect to $\bar{g}$. To study the spectrum of graviton modes, we would like $(\bar{\square}-\lambda) \bar{h}_{\mu \nu}=0$, where $\bar{\square}$ is the $4 \mathrm{D}$ Laplacian with respect to $\bar{g}$ and $\lambda$ is the eigenvalue. The Pauli-Fierz mass of graviton [36, 37] is given by $m^{2}=\lambda-2 k$. By linearizing the field equations, $\psi$ satisfies $[20,23]$

$$
-e^{2 A}\left(\psi^{\prime \prime}+\left(B^{\prime}+4 A^{\prime}\right) \psi^{\prime}+e^{-2 B} \frac{\partial^{2} \psi}{\partial \theta^{2}}\right)=m^{2} \psi,
$$


where the dependence on background fields is hidden in the warp factors. Now we can further decompose $\psi(y)=\bar{\psi}(r) e^{i n \theta}$, where $n$ is like a winding number. Note that in terms of $\Psi=e^{2 A} \psi$, eq. (2.23) can be rewritten into a Schrodinger-like equation:

$$
(-\tilde{\square}+V(y)) \Psi(y)=m^{2} \Psi(y), \quad V(y)=e^{-2 A} \tilde{\square} e^{2 A},
$$

where the Laplacian $\tilde{\square}$ is with respect to the inversely warped internal metric $\tilde{g}=\operatorname{diag}$. $e^{-2 A}\left(1, e^{2 B}\right) . \Psi$ also represents the amplitude of gravity in the extra dimensions. Therefore, we usually refer $\Psi$ and even $\psi$ as wavefunctions, but they are not quantum-mechanical wavefunctions.

When we say the graviton mode is localized, it means that $\Psi$ peaks at $r=0$ only and decays when it goes far from the brane. Its norm is given by

$$
\|\Psi\|^{2}=\int d r d \theta e^{B+2 A}|\psi|^{2} .
$$

To have physical graviton modes, the normalizability is a required boundary condition at infinity. It is non-trivial since we are integrating infinitely large proper radius $r$. There is also a matching condition at the brane position.

Here is its derivation. Using eq. (2.21), we have

$$
K_{\mu \nu}=A^{\prime} e^{2 A}\left(\bar{g}_{\mu \nu}+h_{\mu \nu}\right)+\frac{1}{2} e^{2 A} h_{\mu \nu}^{\prime}
$$

while $K_{\theta \theta}, K$ and $\sqrt{-g}$ remain unchanged by tracelessness of $h$. Therefore the $\theta \theta$-component of eq. (2.17) is unchanged and the $\mu \nu$-component becomes

$$
-\lim _{r \rightarrow 0} \frac{2 \pi}{\kappa^{2}} e^{6 A}\left[\left(e^{B}\left(3 A^{\prime}+B^{\prime}\right)-1\right)\left(\bar{g}_{\mu \nu}+h_{\mu \nu}\right)-\frac{1}{2}\left(e^{B}-r\right) h_{\mu \nu}^{\prime}\right]=e^{2 A} T_{b}\left(\bar{g}_{\mu \nu}+h_{\mu \nu}\right) .
$$

The new matching condition for graviton modes is

$$
\lim _{r \rightarrow 0} e^{6 A}\left(e^{B}-r\right) \psi^{\prime}=0 .
$$

Note that by definition of branes $e^{B}-r \rightarrow 0$ when $r \rightarrow 0$, so a wavefunction with finite $\psi^{\prime}(0)$ automatically satisfies the condition, but it remains interesting to study the case that $\psi^{\prime}(0)$ diverges.

In general the model cannot be solved analytically. To have a sense on what kinds of geometry the solutions describe, below we consider two exactly solvable cases. Both are with a bulk cosmological constant $V=\frac{\Lambda}{\kappa^{2}}$ and without scalar fields i.e. $c=1$ and $U_{b}=0$, but one is with $k, f, B$ turned on only and another is with $A, B$ turned on only. We will solve for their background geometries and (seemingly valid) massless localized graviton modes.

\subsection{Exactly solvable models}

Here we study two examples to demonstrate the features of the above setup. 
With $\boldsymbol{\Lambda}, \boldsymbol{k}, \boldsymbol{f}, \boldsymbol{B}$. Here we set $A$ to be constant, then eq. (2.13) and (2.14) simply mean $\Lambda=f e^{-8 A}+6 k e^{-2 A}$. Requiring $\lim _{r \rightarrow 0} e^{B}=0$, eq. (2.12) becomes

$$
-9 k e^{-2 A}+2 \Lambda+B^{2}+B^{\prime \prime}=0 \Rightarrow B(r)= \begin{cases}\ln \left(\sin \left(\sqrt{2 \Lambda-9 k e^{-2 A}} r\right)\right)+C_{1} & \Lambda>9 k e^{-2 A} / 2 \\ \ln r+C_{1} & \Lambda=9 k e^{-2 A} / 2 \\ \ln \left(\sinh \left(-\sqrt{-2 \Lambda+9 k e^{-2 A}} r\right)\right)+C_{1} & \Lambda<9 k e^{-2 A} / 2\end{cases}
$$

where $C_{1}$ is an integration constant. Since we are studying noncompact extra dimensions, we only accept $\Lambda \leq \frac{9 k e^{-2 A}}{2}$. The matching conditions reproduce the constraint on $B(0)$ in both cases and require

$$
T_{b}=\left\{\begin{array}{ll}
-\frac{2 \pi}{\kappa^{2}}\left(e^{C_{1}}-1\right) & \Lambda=9 k e^{-2 A} / 2 \\
-\frac{2 \pi}{\kappa^{2}}\left(\sqrt{-2 \Lambda+9 k e^{-2 A}} e^{C_{1}}-1\right) & \Lambda<9 k e^{-2 A} / 2
\end{array} .\right.
$$

Therefore this geometry is supported for all $T_{b}<\frac{2 \pi}{\kappa^{2}}$ given the tuning of Maxwell field.

Now we solve for massless graviton modes. We focus on $n=0$. For $\Lambda=\frac{9 k e^{-2 A}}{2}$,

$$
\psi^{\prime \prime}+\frac{1}{r} \psi^{\prime}=0 \Rightarrow \psi(r)=C_{2} \ln r+C_{3},
$$

which is clearly not localized and not normalizable for all $C_{2}$ and $C_{3}$. For $\Lambda<\frac{9 k e^{-2 A}}{2}$, $\psi^{\prime \prime}+\sqrt{-2 \Lambda+9 k e^{-2 A}} \operatorname{coth}\left(\sqrt{-2 \Lambda+9 k e^{-2 A}} r\right) \psi^{\prime}=0 \Rightarrow \psi(r)=C_{2} \ln \left(\tanh \left(\sqrt{\frac{-2 \Lambda+9 k e^{-2 A}}{4}} r\right)\right)+C_{3}$,

which is localized at $r=0$ and is normalizable if and only if $C_{3}=0$ :

$$
\begin{aligned}
\|\Psi\|^{2} & =2 \pi\left|C_{2}\right|^{2} \int_{0}^{\infty} d r \sinh \left(\sqrt{-2 \Lambda+9 k e^{-2 A}} r\right) \ln \left(\tanh \left(\sqrt{\frac{-2 \Lambda+9 k e^{-2 A}}{4} r}\right)\right)^{2} \\
& =10.3354 \frac{\left|C_{2}\right|^{2}}{\sqrt{-2 \Lambda+9 k e^{-2 A}}}<\infty .
\end{aligned}
$$

However, the matching condition reads

$$
\lim _{r \rightarrow 0}\left(e^{B}-r\right) \psi^{\prime}=C_{2}\left(\sqrt{-2 \Lambda+9 k e^{-2 A}} e^{C_{1}}-1\right)=-\frac{\kappa^{2}}{2 \pi} C_{2} T_{b}=0 .
$$

Since $T_{b} \neq 0$, we also have $C_{2}=0$ and there does not exist any massless graviton mode which satisfies all boundary conditions.

With $\boldsymbol{\Lambda}, \boldsymbol{A}, \boldsymbol{B}$. Here we set $f=k=0$ and non-constant $A$. Solving eq. (2.13) and (2.14) and imposing $\lim _{r \rightarrow 0} e^{B}=0$ yields

$$
\begin{aligned}
& A(r)= \begin{cases}\frac{2}{5} \ln r+C_{1} & \Lambda=0 \\
\frac{2}{5} \ln \left(\cosh \left(\sqrt{\frac{-5 \Lambda}{8}} r\right)\right)+C_{1} \quad \Lambda<0\end{cases} \\
& B(r)=\left\{\begin{array}{ll}
-\frac{3}{5} \ln r+C_{2} & \Lambda=0 \\
-\frac{3}{5} \ln \left(\cosh \sqrt{\frac{-5 \Lambda}{8}} r\right)+\ln \left(\sinh \sqrt{\frac{-5 \Lambda}{8}} r\right)+C_{2} & \Lambda<0
\end{array} .\right.
\end{aligned}
$$


These solutions automatically also satisfy eq. (2.12). With the same reason as above we do not consider $\Lambda>0$. However for $\Lambda=0$, eq. (2.19) becomes $e^{4 C_{1}+C_{2}}=0$, which cannot be satisfied. Therefore this geometry is not supported due to matching conditions. Even if it is supported, the wavefunction is not normalizable under this geometry as above.

For $\Lambda<0$, the solution is usually known as the AdS soliton [38]. The matching conditions lead to

$$
T_{b}=-\frac{\pi e^{4 C_{1}}\left(\sqrt{-10 \Lambda} e^{C_{2}}-4\right)}{2 \kappa^{2}} .
$$

Therefore this geometry is valid for all $\Lambda \leq 0$ and $T_{b}$. Now the massless $n=0$ graviton mode satisfies

$$
\psi^{\prime \prime}+\sqrt{\frac{-5 \Lambda}{2}} \operatorname{coth}\left(\sqrt{\frac{-5 \Lambda}{2}} r\right) \psi^{\prime}=0 \Rightarrow \psi(r)=C_{3} \ln \left(\tanh \left(\sqrt{\frac{-5 \Lambda}{8}} r\right)\right)+C_{4} .
$$

This is a more extreme example than above. Even when the warping amplifies the localized wavefunction, it can still be normalized when $C_{4}=0$ :

$$
\begin{aligned}
\|\Psi\|^{2} & =2 \pi\left|C_{3}\right|^{2} \int_{0}^{\infty} d r \sinh \left(\sqrt{\frac{-5 \Lambda}{8}} r\right) \cosh ^{1 / 5}\left(\sqrt{\frac{-5 \Lambda}{8} r}\right) \ln \left(\tanh \left(\sqrt{\frac{-5 \Lambda}{8}} r\right)\right)^{2} \\
& =2.80403 \frac{\left|C_{3}\right|^{2}}{\sqrt{-\Lambda}}<\infty
\end{aligned}
$$

However, the matching condition reads

$$
\lim _{r \rightarrow 0} e^{6 A}\left(e^{B}-r\right) \psi^{\prime}=\frac{C_{3} e^{6 C_{1}}\left(\sqrt{-10 \Lambda} e^{C_{2}}-4\right)}{4}=-\frac{\kappa^{2}}{2 \pi} e^{2 C_{1}} C_{3} T_{b}=0 .
$$

Again, it forces $C_{3}=0$ and there is not any massless graviton modes.

As a remark, there is another interesting solution to this background, which is

$$
A=C_{1}-\sqrt{\frac{-\Lambda}{10}} r, \quad B=C_{2}-\sqrt{\frac{-\Lambda}{10}} r .
$$

This is the only solution with a decaying warp factor $e^{2 A}$, hence the constant wavefunction can be localized and normalized. On the other hand, it does not satisfy $\lim _{r \rightarrow 0} e^{B}=0$. Notice that the ordinary type-II Randall-Sundrum (RS-II) model [24] is equivalent to the constant wavefunction in our formulation. This is why we cannot construct such model with two noncompact extra dimensions. However, we will see that this solution has another related implication when combined with compactification.

\subsection{Necessary conditions for existence of massless localized graviton}

We already see that localized $\psi$ is rejected by the matching condition in the above examples. Here we consider back the general setup in section 2.1 and prove the necessary conditions in order to localize a massless graviton. 
Consider the wavefunction of a massless graviton. By separation of variables, $\psi(y)=$ $\bar{\psi}(r) e^{i n \theta}$ where $n$ is integer. We have that $\bar{\psi}(0) e^{i n \theta}$ are equal for all $\theta$, which means $\bar{\psi}(0)=0$ and the graviton mode cannot be localized when $n \neq 0$. Therefore we only consider $n=0$. Eq. (2.23) implies

$$
\psi^{\prime \prime}+\left(B^{\prime}+4 A^{\prime}\right) \psi^{\prime}=0 \Rightarrow \psi^{\prime}=C e^{-B-4 A},
$$

where $C$ is an integration constant. Using the fact that $A(0)$ is finite in order to have physical $4 \mathrm{D}$ induced metric, the matching condition eq. (2.28) reads,

$$
\lim _{r \rightarrow 0} C\left(1-\frac{r}{e^{B}}\right)=0 .
$$

There is a subtlety that there are two ways to understand the matching condition. The first one is to note that since the matching condition is obtained by integrating a small circle around brane position, when we say $r \rightarrow 0$ we actually mean substituting some small $r=\epsilon$. Since in curved background $\frac{\epsilon}{e^{B(\epsilon)}} \neq 1$, the matching condition simply implies $C=0$ i.e. constant wavefunction. This is actually an intuitive statement since there is no brane sources to drive the massless graviton modes.

The second one is to directly take the $r \rightarrow 0$ limit. We then need to be careful what the ratio $\frac{r}{e^{B}}$ contributes. Note that this ratio captures the defect angle at brane position. To be precise, the defect angle $\delta=2 \pi(1-\alpha)$ is given by

$$
\alpha=\lim _{r \rightarrow 0} \frac{e^{B}}{r} .
$$

Therefore the matching condition implies that $C=0$ unless there is no defect angle, which is not true for typical brane sources. We thus conclude that $\psi$ must be a constant. If the warp factor $e^{B+2 A}$ does not vanish at infinity, the constant wavefunction is not normalizable and there is no massless graviton modes. Therefore we state:

Massless localized graviton exists only when $\int d r d \theta e^{B+2 A}$ is finite.

\subsection{No-go theorems}

Two noncompact extra dimensions. The constant wavefunction can only be localized and normalized by the warp factor $e^{2 A}$. Through this, we show that within our model, the constant wavefunction is not a valid solution.

First, we assume that $e^{A}$ is decreasing to small values at large $r$, hence $A$ is decreasing at large $r$. By combining eq. (2.13) and (2.14), for sufficiently large $r>r_{1}$ we have

$$
A^{\prime \prime}+A^{\prime 2}=A^{\prime} B^{\prime}-\frac{\kappa^{2}}{4} \phi^{2}<0,
$$

since $B^{\prime}>0$ at large $r$. It means that $\left(e^{A}\right)^{\prime \prime}=e^{A}\left(A^{\prime \prime}+A^{\prime 2}\right)<0$ for $r>r_{1}$. Then for all $y>x>r_{1}$, if $x, y$ are within the domain of $A$, by Mean Value theorem there exists $\xi \in(x, y)$ such that

$$
\frac{e^{A(y)}-e^{A(x)}}{y-x}=\left(e^{A}\right)^{\prime}(\xi) \leq\left(e^{A}\right)^{\prime}(x) \Rightarrow e^{A(y)} \leq e^{A(x)}+\left(e^{A}\right)^{\prime}(x)(y-x) .
$$


Since $\left(e^{A}\right)^{\prime}(x)<0$, by choosing sufficiently large $y$ such that $e^{A(x)}+\left(e^{A}\right)^{\prime}(x)(y-x)<0$, eq. (2.46) means that $e^{A}$ must vanish at some finite $r<y$, but not infinity. This implies that there is no solution at infinity, which violates the assumption of noncompact extra dimensions. By contradiction, it means that $e^{A}$ does not vanish at infinity in noncompact extra dimensions, thus cannot localize or normalize the constant wavefunction.

This finishes the proof of the no-go theorem on localized gravity around codimension-2 branes in two noncompact extra dimensions. However as we will see, this proof makes use of unique features in codimension-2 models, and cannot be generalized to higher codimensions.

One compact and one noncompact extra dimensions. So far we have studied the possibility of localized gravity with extra dimensions being noncompact in two directions i.e. $B \rightarrow+\infty$. It still remains interesting to explore the case when $B$ keeps finite, such that at long distances the spacetime looks like with one noncompact extra dimension, but with another one compactified dimension. For example, we can attach a small circle at each point in the Randall-Sundrum model, such that the extra dimensions have topology $\mathbb{R} \times S^{1}$, and the geometry looks like a long thin tube. In such cases, at long distances we cannot really tell whether the brane is codimension-1 or codimension- 2 .

If the brane is still codimension-1, that means the brane wraps around the hidden circle. Such geometry actually can be easily obtained, such as in eq. (2.41). We identify $r$ as the height of the cylinder and $e^{B}$ as the compactification radius. We further replace $r$ by $|r|$ in those equations. The compact extra dimension becomes smaller and smaller as $r$ increases, so by fixing suitable $C_{2}$ we can keep the compact extra dimension very small for all $r$. Now if we put a wrapped 4 -brane at $r=0$, with appropriate matching conditions the constant massless graviton wavefunction is localized around the circle $r=0$ by the warp factor $A$. It is equivalent to the ordinary RS-II model [24] but in two extra dimensions with one being compactified.

If the brane is actually codimension-2, at $r=0$ the hidden dimension must shrink to a point, in order to maintain the symmetry of the system. This means that the topology of the bulk is changed, and it is non-trivial whether the long distance physics remain the same. Below we provide a non-rigorous argument to show that it is not possible to both achieve such geometry and localize gravity.

Again we first assume that localized gravity in such geometry is possible. We start with the behavior of $A$ and $B$. In this case we still have $\lim _{r \rightarrow 0} e^{B}=0$ i.e. $B(0) \rightarrow-\infty$. Since at $r=0$ we have $\left(e^{B}\right)^{\prime}>0$, it means $B^{\prime}(0) \rightarrow+\infty$. To localize gravity at brane position, we want $A^{\prime}(0)$ to be negative. Then by eq. (2.45), we get $A^{\prime \prime}(0) \rightarrow-\infty$. In particular it means that $A^{\prime}(0)$ needs to be finite. Eq. (2.19) then tells $U_{b}=0$, which implies $\frac{\partial T_{b}}{\partial \phi}=0$ by eq. (2.20).

An increasing $B$ has already been forbidden in last subsection. Therefore, $B$ must turn decreasing at some finite $r$. After $B$ starts decreasing, it must fall not too fast such that $e^{B}$ does not vanish at some non-zero and finite $r$. This can be judged by combining eq. (2.12) to (2.14), which gives

$$
e^{-B}\left(e^{B}\right)^{\prime \prime}=B^{\prime \prime}+B^{\prime 2}=-4 A^{\prime} B^{\prime}-\frac{3}{2} f e^{-8 A}-\frac{\kappa^{2}}{2} V .
$$


Now we consider the behavior of the potential $V$. From eq. (2.13), we observe that

$$
V(r=0)=\frac{1}{\kappa^{2}}\left(-6 A^{\prime 2}-4 A^{\prime} B^{\prime}+f e^{-8 A}+6 k e^{-2 A}\right)+\left.\frac{1}{2} \phi^{\prime 2}\right|_{r=0} \rightarrow+\infty .
$$

If $V$ keeps positive for all $r$, after $B$ starts decreasing at some finite $r$, we have $\left(e^{B}\right)^{\prime \prime}<0$ and $e^{B}$ vanishes at another finite $r$ by the argument in last subsection. Therefore to meet the above requirement $V$ must turn negative at some finite $r=r_{2}$. It means that there is always a region of small $r$ between 0 and $r_{2}$ that has negative $V^{\prime}$ with large magnitude. In particular, there must be a point where $V^{\prime}$ diverges to minus infinity, since $V$ starts at positive infinity.

We then turn to the field equation for the scalar field i.e. eq. (2.8). Multiplying it with $\phi^{\prime}$, we get

$$
\left(\frac{1}{2} \phi^{\prime 2}\right)^{\prime}+\left(B^{\prime}+4 A^{\prime}\right) \phi^{\prime 2}=V^{\prime}+\frac{1}{4} c^{\prime} \frac{f}{c} e^{-8 A} .
$$

At small $r$ and smaller than $r_{2}, B^{\prime}+4 A^{\prime}$ is positive while the $f$ term is suppressed by large $e^{A}$. Therefore in that region the kinetic energy $\frac{1}{2} \phi^{\prime 2}$ decreases rapidly, and even infinitely fast at the point where $V^{\prime}$ diverges. However, since $\frac{1}{2} \phi^{\prime 2}$ is always positive, it means that $\phi^{\prime}$ must start at very large value at $r=0$. By integrating eq. (2.49), we see that $\phi^{\prime}$ actually starts at infinity, which implies $\frac{\partial T_{b}}{\partial \phi} \neq 0$ by eq. (2.15). Note that we now have a contradiction, so we can conclude that we cannot both obtain a long thin tube geometry and localize gravity around a codimension-2 brane in that geometry. Notice that here we have used many properties specific to two extra dimensions, and the proof cannot be generalized to more than one compact dimensions.

In conclusion, within our setup, whenever we see a codimension-1 model but with a hidden compact dimension, we can claim that the brane must also wrap that dimension i.e. it must be also codimension-1 but not codimension-2. The only difference between two scenarios is the topology of the extra dimensions. This result reveals the typical behavior that the topological details of compactified extra dimensions can affect the low-energy physics, even when we cannot observe those dimensions.

\section{Branes with higher codimensions}

In this section, we investigate the no-go theorem in codimensions higher than two.

\subsection{Klebanov-Strassler throat}

After the above proof, it is natural to consider whether localization of gravity in more than two noncompact extra dimensions is still possible. In particular, such possibility in string theory solution is important to string phenomenology. Here as an example, we consider the Klebanov-Strassler throat [30]. Similarly to above, we focus on the UV region, but not the deformed IR region of the throat. Therefore for simplicity, we only consider the undeformed version of the throat i.e. the geometry in [31], and similar but more complicated arguments should hold for that in [30]. The metric is given by

$$
d s_{10}^{2}=e^{2 a(u)-5 q(u)} \eta_{\mu \nu} d x^{\mu} d x^{\nu}+e^{-5 q(u)} d u^{2}+e^{3 q(u)} d s_{T^{1,1}}^{2} .
$$


Here the functions $a$ and $q$ are analog to $A$ and $B$ in our codimension- 2 context, $u$ is the radial coordinate of the throat and $T^{1,1}=(\mathrm{SU}(2) \times \mathrm{SU}(2)) / \mathrm{U}(1)$ is the base of the conifold with metric

$$
d s_{T^{1,1}}^{2}=\frac{1}{9}\left(d \psi+\sum_{i=1}^{2} \cos \theta_{i} d \phi_{i}\right)^{2}+\frac{1}{6} \sum_{i=1}^{2}\left(d \theta_{i}^{2}+\sin ^{2} \theta_{i} d \phi_{i}^{2}\right) .
$$

The norm of constant wavefunction is given by

$$
\|\Psi\|^{2}=\int d^{6} x \sqrt{e^{-5 q}\left(e^{3 q}\right)^{5}} e^{2 a-5 q} \propto \int d u e^{2 a} .
$$

Therefore to study the normalizability, we study whether $e^{2 a}$ can decay to zero at infinity. By solving the type IIB supergravity equations, we have

$$
a(u)=A_{0}+q(u)+\frac{1}{P} T(u),
$$

where the constant $P>0$ and $T(u)$ are related to the R-R 3-form field strength and NS-NS 2 -form potential respectively, $A_{0}$ is an integration constant. There is another solution with $a=A_{0}+u$, which is clearly not normalizable. Define $Y=e^{6 q}$ and $K=4+P T$ ( $K$ is actually related to the self-dual 5 -form field strength), the field equations give

$$
K^{\prime}=P^{2} Y^{-2 / 3}, \quad \frac{d Y}{d K}=\frac{1}{P^{2}}(4 Y-K),
$$

where here "prime" represents derivative with respect to $u$. There is a general solution for $Y$ :

$$
Y=a_{0} e^{4 K / P^{2}}+\frac{K}{4}+\frac{P^{2}}{16}
$$

where $a_{0}$ is an integration constant. Note that $K$ is always increasing, so as $T$. If $a_{0} \geq 0$, $Y$ is also increasing, thus $a$ is increasing and $e^{2 a}$ cannot decay to zero at infinity. Now we focus on the case with $a_{0}<0$ and $Y$ is not always increasing. It turns out that this case violates the assumption of noncompact extra dimensions.

The proof is as follows. Let $u=u_{1}$ be a point such that $Y$ is strictly decreasing. For all $u>u_{1}$ and within domain of $Y$,

$$
Y^{\prime}=\frac{d Y}{d K} K^{\prime}=Y^{-2 / 3}\left(4 a_{0} e^{4 K(u) / P^{2}}+\frac{P^{2}}{4}\right) \leq Y^{-2 / 3}\left(4 a_{0} e^{4 K\left(u_{1}\right) / P^{2}}+\frac{P^{2}}{4}\right) \leq 0,
$$

and

$$
K^{\prime \prime}=\frac{d\left(K^{\prime}\right)}{d Y} Y^{\prime}=-\frac{2}{3} P^{2} Y^{-5 / 3} Y^{\prime} \geq 0 .
$$

Therefore $K^{\prime} \geq 0$ and $K^{\prime \prime} \geq 0$ for all such $u$. Now for all $y>x>u_{1}$, if $x, y$ are within the domain of $A$, by Mean Value theorem there exists $\xi \in(x, y)$ such that

$$
\frac{K(y)-K(x)}{y-x}=K^{\prime}(\xi) \geq K^{\prime}(x) \Rightarrow K(y) \geq K(x)+K^{\prime}(x)(y-x) .
$$

Let $Y=0$ at finite $K=K_{1}$. By choosing sufficiently large $y$, eq. (3.9) guarantees that $K$ must reach $K_{1}$ at some finite $u=u_{2}$. In other words, $Y$ decreases to zero at $u_{2}$ and 
there is no solution for $u>u_{2}$. Furthermore, numerical integration shows that the proper radius $\int^{u_{2}} d u e^{-5 q / 2}=\int{ }^{u_{2}} d u Y^{-5 / 12}$ is finite. Therefore we conclude that $Y$ can only be increasing in noncompact extra dimensions, and constant wavefunction is not normalizable. Therefore, the only way to localize gravity with the Klebanov-Strassler throat is to make a UV cutoff of the throat, as how the throat is attached to a compact manifold in the context of compactification.

\subsection{General setup}

Before we prove the no-go theorem in general, let us first rewrite some of the general setup in section 2.1 for codimension- $d$ branes where $d \geq 3$. We let the bulk Lagrangian be

$$
S_{\text {bulk }}=-\int d^{4} x d^{d} y \sqrt{-g}\left(\frac{1}{2 \kappa^{2}} R+\frac{1}{2} \partial^{M} \phi \partial_{M} \phi+\sum_{p} \frac{1}{2 p} c_{p}(\phi) F_{p}^{2}+V(\phi)\right)+S_{\mathrm{GH}},
$$

where $2 \leq p \leq d$ and $F_{p}=d A_{p-1}$ is a $p$-form field strength. We use the notation $F_{p}^{2}=$ $F_{M_{1} M_{2} \ldots M_{p}} F^{M_{1} M_{2} \ldots M_{p}}$. We first study the case where all extra dimensions are noncompact. The metric becomes

$$
d s^{2}=g_{M N} d x^{M} d x^{N}=e^{2 A(r)} \bar{g}_{\mu \nu} d x^{\mu} d x^{\nu}+d r^{2}+e^{2 B(r)} \hat{g}_{a b}(\theta) d \theta^{a} d \theta^{b},
$$

where $1 \leq a, b \leq d-1$ and $\hat{g}$ is the metric for internal angular coordinates. By symmetry $A_{p-1}$ depends on $r$ only, and the non-zero components of $F_{p}$ are

$$
F_{r a_{1} a_{2} \ldots}, \quad F_{\mu \nu \rho \sigma r a_{1} a_{2} \ldots} \propto \epsilon_{\mu \nu \rho \sigma} .
$$

Here $a_{1}, a_{2}, \ldots$ are angular coordinates and $\epsilon_{\mu \nu \rho \sigma}$ is the Levi-Civita tensor in brane directions. The latter exists only when $p \geq 5$. Such field strengths do appear in, for example, type IIB string theory.

Let us now consider the brane-bulk matching conditions. In higher codimensions, it is natural to generalize eq. (2.18) to

$$
-\lim _{r \rightarrow 0} \frac{2 \pi^{d / 2}}{\Gamma(d / 2) \kappa^{2}} e^{(d-1) B+4 A}\left(3 A^{\prime}+(d-1) B^{\prime}\right)=T_{b}, \quad(\mu \nu)
$$

and the boundary condition for $\psi^{\prime}$ is

$$
\lim _{r \rightarrow 0} e^{6 A}\left(e^{(d-1) B}-r^{d-1}\right) \psi^{\prime}=0 .
$$

The graviton mode equation is [20,23]

$$
-e^{2 A}\left(\psi^{\prime \prime}+\left((d-1) B^{\prime}+(d+2) A^{\prime}\right) \psi^{\prime}+e^{-2 B} \hat{\square} \psi\right)=m^{2} \psi,
$$

where $\hat{\square}$ is the Laplacian with respect to $\hat{g}$. As in section 2.3, massless localized $\psi$ only depends on $r$, then we have $\psi^{\prime}=C e^{-(d-1) B-(d+2) A}$ where $C$ is the integration constant, the boundary condition becomes

$$
\lim _{r \rightarrow 0} C\left(1-\left(\frac{r}{e^{B}}\right)^{d-1}\right)=0
$$


which is satisfied in curved background only if $C=0$ i.e. $\psi$ is constant. Therefore the statement in section 2.3 also holds in higher codimensions. The norm of $\psi$ is now defined as [23]

$$
\|\Psi\|^{2}=\int d r \prod_{i} d \theta^{i} \sqrt{\hat{g}} e^{(d-1) B+2 A}|\psi|^{2} .
$$

In order to have normalizable solutions, we thus require $e^{(d-1) B+2 A}$ to vanish at infinity.

We next study the field equations. Those for the Maxwell fields are

$$
\nabla_{M}\left(c_{p} F^{M N_{1} N_{2} \ldots N_{p-1}}\right)=0
$$

These yield schematically,

$$
c_{p} F_{p}^{2}=\frac{f_{1 p}^{2}}{c_{p}} e^{-2(d-p) B-8 A}+\frac{f_{2 p}^{2}}{c_{p}} e^{-2(d-p+4) B},
$$

where $f_{1 p}$ and $f_{2 p}$ are some finite functions of the coordinates other than $r$. They are contributed by the former and the latter in eq. (3.12) respectively. At large $r$, the $f_{2 p}$ term is suppressed by large $e^{B}$, so we can safely ignore it for our purpose as long as $f_{1 p} \neq 0$. Below we just assume $c_{p} F_{p}^{2} \propto c_{p}^{-1} e^{-2(d-p) B-8 A}$. Next we consider the $a b$-components of the Einstein's equation:

$$
\begin{aligned}
g_{a b} & \left(10 A^{\prime 2}+4(d-2) A^{\prime} B^{\prime}+4 A^{\prime \prime}+\frac{(d-1)(d-2)}{2} B^{\prime 2}+(d-2) B^{\prime \prime}-6 k e^{-2 A}\right)-\hat{R}_{a b}+\frac{1}{2} \hat{R} \hat{g}_{a b} \\
& =\sum_{p} c_{p} \kappa^{2}\left(F_{a X_{1} X_{2} \ldots X_{p-1}} F_{b} X_{1} X_{2} \ldots X_{p-1}-\frac{1}{2 p} g_{a b} F_{p}^{2}\right)-\kappa^{2} g_{a b}\left(\frac{1}{2} \phi^{\prime 2}+V\right),
\end{aligned}
$$

where $a, b$ label the angular coordinates and $X$ labels all internal coordinates. $\hat{R}_{a b}$ and $\hat{R}$ are the Ricci tensor and Ricci scalar with respect to $\hat{g}$ respectively. We expect $\hat{R}$ to be a finite function of $\theta$. We then take a partial trace i.e. contracting eq. (3.20) with $g^{a b}$ to reach

$$
\begin{aligned}
& (d-1)\left(10 A^{\prime 2}+4(d-2) A^{\prime} B^{\prime}+4 A^{\prime \prime}+\frac{(d-1)(d-2)}{2} B^{\prime 2}+(d-2) B^{\prime \prime}\right) \\
& \quad=(d-1)\left(6 k e^{-2 A}-\kappa^{2}\left(\frac{1}{2} \phi^{\prime 2}+V\right)\right)+\sum_{p} c_{p} \kappa^{2}\left(1-\frac{d+1}{2 p}\right) F_{p}^{2}-\frac{d-3}{2} \hat{R} e^{-2 B}=-J,
\end{aligned}
$$

where we have used $F_{a X_{1} X_{2} \ldots X_{p-1}} F^{a X_{1} X_{2} \ldots X_{p-1}}=\left(1-\frac{1}{p}\right) F_{p}^{2}$. We have also defined a quantity $J$ which is useful later. Similarly, the $r r$-component is

$$
\begin{aligned}
6 A^{\prime 2} & +4(d-1) A^{\prime} B^{\prime}+\frac{(d-1)(d-2)}{2} B^{\prime 2} \\
& =6 k e^{-2 A}-\kappa^{2}\left(-\frac{1}{2} \phi^{\prime 2}+V\right)+\sum_{p} c_{p} \kappa^{2} \frac{1}{2 p} F_{p}^{2}-\frac{1}{2} \hat{R} e^{-2 B}
\end{aligned}
$$




\subsection{No-go theorem}

Now we show that the constant graviton wavefunction is not a valid solution, similarly to section 2.4. First we try to derive an equation analogous to eq. (2.45). By combining eq. (3.21) and (3.22), we have

$$
(d-1)\left(4 A^{\prime 2}-4 A^{\prime} B^{\prime}+4 A^{\prime \prime}+(d-2) B^{\prime \prime}\right)=-(d-1) \kappa^{2} \phi^{2}+\sum_{p} c_{p} \kappa^{2}\left(1-\frac{d}{p}\right) F_{p}^{2}+\hat{R} e^{-2 B} .
$$

The main obstruction of proving a similar no-go theorem as in section 2.4 is the $B^{\prime \prime}$ term, which does not appear for codimension-2 branes. Although it is not typical, noncompact extra dimensions do not stop $B^{\prime \prime}$ from being negative with large magnitude. The $\hat{R}$ term can also be positive. Therefore, we will prove the no-go theorem in a different way with some additional assumptions. The assumptions are

- There is always a form field background with $f_{1 p}^{2}>0$ for some $p$ and nonvanishing couplings. Mathematically, it means that $c_{p}$ is bounded from above, so $f_{1 p}^{2} / c_{p}$ is bounded from below by a positive number.

or

- The spacetime in brane directions is curved i.e. $k \neq 0$.

Such background is common in string theory (motivated) setups. We also assume

- Some of the terms are dominant in $J$ at large $r$. In other words, $J$ does not oscillate between positive and negative values at large $r$. It enables us to use arguments similar to the proof of Maldacena-Nunez no-go theorem [39] on existence of dS compactifications.

Here is an outline of the proof:

- We first assume that $e^{(d-1) B+2 A}$ is decreasing to small values at large $r$, hence $A$ is also decreasing at large $r$. We then prove that for all backgrounds following the above assumptions, $e^{(d-2) B / 2+2 A}$, and thus $e^{(d-1) B+2 A}$ can decrease to zero only at finite $r$, but not infinity. As in section 2.4, it means that the constant wavefunction cannot be localized or normalized. We divide the backgrounds into several cases:

- The dominant term in $J$ at large $r$ is positive: we construct upper bounds of $e^{(d-2) B / 2+2 A}$ by concavity to show that it must vanish at some finite $r$ instead of infinity.

- The dominant term in $J$ at large $r$ is negative: the form of the possible dominant terms and the assumptions give a differential inequality. It again leads to upper bounds of $e^{(d-2) B / 2+2 A}$ showing that it must vanish at some finite $r$ instead of infinity.

- There may be more than one terms that are as dominant as each other. This only modifies the numerical coefficient of the asymptotic form of $J$, which does not change the above arguments.

- Combining the above cases, we can conclude for all the above backgrounds, the constant wavefunction is not a valid solution. 
Let us now fill in the details of the proof.

- The dominant term in $J$ at large $r$ is positive:

We have $J>0$ at large $r$. Note that we can rewrite the left hand side of eq. (3.21) into

$$
\begin{aligned}
& (d-1)\left(10 A^{\prime 2}+4(d-2) A^{\prime} B^{\prime}+4 A^{\prime \prime}+\frac{(d-1)(d-2)}{2} B^{\prime 2}+(d-2) B^{\prime \prime}\right) \\
& =(d-1)\left(2 A^{\prime 2}+\frac{d-2}{2} B^{\prime 2}+2\left(\frac{d-2}{2} B^{\prime \prime}+2 A^{\prime \prime}+\left(\frac{d-2}{2} B^{\prime}+2 A^{\prime}\right)^{2}\right)\right) \\
& =(d-1)\left(2 A^{\prime 2}+\frac{d-2}{2} B^{\prime 2}+2 e^{-(d-2) B / 2-2 A}\left(e^{(d-2) B / 2+2 A}\right)^{\prime \prime}\right) .
\end{aligned}
$$

Therefore $\left(e^{(d-2) B / 2+2 A}\right)^{\prime \prime}$ is negative at large $r$ and $e^{(d-2) B / 2+2 A}$ cannot vanish at infinity, according to the arguments in section 2.4.

- The dominant term in $J$ at large $r$ is negative:

We first stick with the first assumption on form fields. From eq. (3.23),

$$
(d-1)\left(-4 A^{\prime} B^{\prime}+4 A^{\prime \prime}+(d-2) B^{\prime \prime}\right) \leq \hat{R} e^{-2 B} .
$$

Now there is a negative term $\propto e^{-2(d-p) B-8 A}$ in $J$. No matter whether that term is dominant in $J$, there must exist a constant $v_{0}>0$ such that $-J-\hat{R} e^{-2 B} \geq v_{0} e^{-2(d-p) B-8 A} \geq$ $v_{0} e^{-2(d-2) B-8 A}$ at large $r$. Then from eq. (3.21), at large $r$ we have

$$
\begin{aligned}
& (d-1)\left(10 A^{\prime 2}+4(d-1) A^{\prime} B^{\prime}+\frac{(d-1)(d-2)}{2} B^{\prime 2}\right) \\
& =-J-(d-1)\left(-4 A^{\prime} B^{\prime}+4 A^{\prime \prime}+(d-2) B^{\prime \prime}\right) \geq v_{0} e^{-2(d-2) B-8 A} .
\end{aligned}
$$

In addition, since $e^{(d-1) B+2 A}$ is decreasing at large $r$, we have $(d-1) A^{\prime} B^{\prime}+2 A^{\prime 2} \geq 0$ at large $r$. Straightforward computation then leads to

$$
\begin{gathered}
\frac{2(d-1)}{\min \{4, d-2\}}\left(\frac{d-2}{2} B^{\prime}+2 A^{\prime}\right)^{2} \\
\quad= \begin{cases}8 \frac{d-1}{d-2} A^{\prime 2}+4(d-1) A^{\prime} B^{\prime}+\frac{(d-1)(d-2)}{2} B^{\prime 2} & d \leq 6 \\
10 A^{\prime 2}+4(d-1) A^{\prime} B^{\prime}+\frac{(d-1)(d-2)^{2}}{8} B^{\prime 2}+(d-6)\left((d-1) A^{\prime} B^{\prime}+2 A^{\prime 2}\right) & d \geq 6\end{cases} \\
\geq 10 A^{\prime 2}+4(d-1) A^{\prime} B^{\prime}+\frac{(d-1)(d-2)}{2} B^{\prime 2} .
\end{gathered}
$$

We then reach

$$
\left(\frac{d-2}{2} B^{\prime}+2 A^{\prime}\right)^{2} \geq v e^{-4((d-2) B / 2+2 A)},
$$

where $v=v_{0} \frac{\min \{4, d-2\}}{2(d-1)^{2}}$ is another positive constant. Since $e^{(d-2) B / 2+2 A}$ is decreasing at large $r$, the above can be simplified to

$$
\left(e^{(d-2) B+4 A}\right)^{\prime} \leq-2 \sqrt{v} .
$$


Therefore by Mean Value theorem, $e^{(d-2) B / 2+2 A} \leq(C-2 \sqrt{v} r)^{1 / 2}$ for some constant $C$ at large $r$. Again it means that $e^{(d-2) B / 2+2 A}$ must vanish at some finite $r$.

If we are under the second assumption on brane curvature, we can just change $v_{0} e^{-2(d-2) B / 2-8 A}$ to $v_{0} e^{-2 A}$ in eq. (3.26). Since we have $e^{-2 A} \geq e^{-(d-2) B / 2-2 A}$ at large $r$, the same proof with different numerical coefficients applies.

Finally, we consider a bulk which is product of $d_{c}$ compact extra dimensions and $d_{n c} \geq 2$ noncompact extra dimensions. The brane has codimension $\left(d_{c}+d_{n c}\right)$. Schematically, let the metric be

$$
d s^{2}=e^{2 A(r)} \bar{g}_{\mu \nu} d x^{\mu} d x^{\nu}+d r^{2}+e^{2 B(r)} \hat{g}_{a b}(\theta) d \theta^{a} d \theta^{b}+e^{2 D(r)} d s_{C}^{2},
$$

where $d s_{C}^{2}$ is the metric of the compact extra dimensions, which does not depend on $x, r, \theta$. Now $a, b$ run from 1 to $\left(d_{n c}-1\right)$. To specify the compactness, we let $e^{2 D}$ be finite and small for all $r$, while $e^{2 B}$ diverges to infinity. It is natural that $D$ only contributes to the field equations subdominantly at large $r$ when comparing to $B$. Therefore the large $r$ behavior of the system is not affected and the above proof still holds. This finishes the proof of the desired no-go theorem.

\section{Conclusion}

We have studied the invalidity of the brane world scenario with noncompact extra dimensions when compared to our observation of four-dimensional gravity. That is, we show that localization of gravity around the brane is not achievable within our general model, which is codimension-2 branes in at least one noncompact extra dimension, or higher codimensional branes in at least two noncompact extra dimensions. We therefore conclude that compactification is necessary to build consistent extra dimensions in such setup.

Below we add some remarks to our results and point out some future directions:

- In literature, we already know that noncompact extra dimensions are usually not favorable for phenomenology. The point of our result is that we give quantitative statements to formally exclude the possibility of localized gravity in certain setups. We give explicit sufficient conditions for the no-go theorems to hold. Therefore, the no-go theorems are still more robust than the implicit statement in literature.

- We have proved the no-go theorem in a quite general context, but we are not claiming that localization of gravity with two or more noncompact extra dimensions must be impossible. It is easy to go beyond the no-go theorem by, for example, adding higher-derivative terms into the action and field equations, or consider different kinds of background such as that with only the scalar field. Indeed, in string theory a $R^{4}$ coupling in noncompact bulk can induce 4D Einstein gravity on the brane [40]. This is a clear counterexample of the no-go theorem. On the other hand, we are not saying that localization of gravity becomes easy outside our conditions in the no-go theorem. It remains interesting to understand how general the no-go theorem can be. 
- We also exclude the case of codimension-2 branes with one compact and one noncompact extra dimensions. The proof involves many properties appear only in codimension-2 models, and it is non-trivial whether the cases with more compact extra dimensions are also excluded. A more general proof is therefore needed to complement our no-go theorem.

- In the proof of our results, the techniques we used are almost purely mathematical, except applying some basic physical properties of the system. To understand more the models, a physical interpretation, or even derivation of our results is needed. Especially, we should have a clear understanding on how the distinct dynamics at brane position between codimension-1 and higher-codimension models, which is introduced in section 1, physically abandon the possibility of localized gravity. Surely symmetries play a crucial role in our derivations, but it may be possible to construct the no-go theorem by arguments based on only symmetries and topologies of the system, but not referring to the explicit dynamics of the system.

We hope to address some of the above issues in our further studies.

\section{Acknowledgments}

The author thanks Cliff Burgess for initiating this work and initial collaboration. He thanks Raman Sundrum, Andrew Tolley and Henry Tye for useful discussions. He also thanks Ignatios Antoniadis for pointing out their work as a counterexample. The bulk of this work was done when the author visited Perimeter Institute. Research at Perimeter Institute is supported in part by the Government of Canada through the Department of Innovation, Science and Economic Development Canada and by the Province of Ontario through the Ministry of Economic Development, Job Creation and Trade.

Open Access. This article is distributed under the terms of the Creative Commons Attribution License (CC-BY 4.0), which permits any use, distribution and reproduction in any medium, provided the original author(s) and source are credited.

\section{References}

[1] C.P. Burgess, R.C. Myers and F. Quevedo, A Naturally small cosmological constant on the brane?, Phys. Lett. B 495 (2000) 384 [hep-th/9911164] [InSPIRE].

[2] N. Arkani-Hamed, S. Dimopoulos, N. Kaloper and R. Sundrum, A Small cosmological constant from a large extra dimension, Phys. Lett. B 480 (2000) 193 [hep-th/0001197] [INSPIRE].

[3] J.-W. Chen, M.A. Luty and E. Ponton, A Critical cosmological constant from millimeter extra dimensions, JHEP 09 (2000) 012 [hep-th/0003067] [INSPIRE].

[4] S. Kachru, M.B. Schulz and E. Silverstein, Selftuning flat domain walls in $5 D$ gravity and string theory, Phys. Rev. D 62 (2000) 045021 [hep-th/0001206] [INSPIRE]. 
[5] S. Dimopoulos, S. Kachru, N. Kaloper, A.E. Lawrence and E. Silverstein, Small numbers from tunneling between brane throats, Phys. Rev. D 64 (2001) 121702 [hep-th/0104239] [INSPIRE].

[6] Y. Aghababaie, C.P. Burgess, S.L. Parameswaran and F. Quevedo, Towards a naturally small cosmological constant from branes in 6 D supergravity, Nucl. Phys. B 680 (2004) 389 [hep-th/0304256] [INSPIRE].

[7] C.P. Burgess, P. Grenier and D. Hoover, Quintessentially flat scalar potentials, JCAP 03 (2004) 008 [hep-ph/0308252] [INSPIRE].

[8] S.M. Carroll and M.M. Guica, Sidestepping the cosmological constant with football shaped extra dimensions, hep-th/0302067 [INSPIRE].

[9] I. Navarro, Codimension two compactifications and the cosmological constant problem, JCAP 09 (2003) 004 [hep-th/0302129] [INSPIRE].

[10] C.P. Burgess, Towards a natural theory of dark energy: Supersymmetric large extra dimensions, AIP Conf. Proc. 743 (2004) 417 [hep-th/0411140] [INSPIRE].

[11] C.P. Burgess, Supersymmetric large extra dimensions and the cosmological constant: An Update, Annals Phys. 313 (2004) 283 [hep-th/0402200] [INSPIRE].

[12] C.P. Burgess and L. van Nierop, Technically Natural Cosmological Constant From Supersymmetric 6D Brane Backreaction, Phys. Dark Univ. 2 (2013) 1 [arXiv:1108.0345] [INSPIRE].

[13] C. Charmousis, E. Kiritsis and F. Nitti, Holographic self-tuning of the cosmological constant, JHEP 09 (2017) 031 [arXiv:1704.05075] [INSPIRE].

[14] L. Randall and R. Sundrum, A Large mass hierarchy from a small extra dimension, Phys. Rev. Lett. 83 (1999) 3370 [hep-ph/9905221] [INSPIRE].

[15] T. Gherghetta and A. Pomarol, Bulk fields and supersymmetry in a slice of AdS, Nucl. Phys. B 586 (2000) 141 [hep-ph/0003129] [INSPIRE].

[16] C.A. Scrucca, M. Serone, L. Silvestrini and A. Wulzer, Gauge Higgs unification in orbifold models, JHEP 02 (2004) 049 [hep-th/0312267] [INSPIRE].

[17] E. Dudas, C. Papineau and V.A. Rubakov, Flowing to four dimensions, JHEP 03 (2006) 085 [hep-th/0512276] [INSPIRE].

[18] M. Sakamoto and K. Takenaga, Large gauge hierarchy in gauge-Higgs unification, Phys. Rev. D 75 (2007) 045015 [hep-th/0609067] [INSPIRE].

[19] C.P. Burgess, C. de Rham and L. van Nierop, The Hierarchy Problem and the Self-Localized Higgs, JHEP 08 (2008) 061 [arXiv:0802.4221] [inSPIRE].

[20] C. Csáki, J. Erlich, T.J. Hollowood and Y. Shirman, Universal aspects of gravity localized on thick branes, Nucl. Phys. B 581 (2000) 309 [hep-th/0001033] [InSPIRE].

[21] I. Oda, Locally localized gravity models in higher dimensions, Phys. Rev. D 64 (2001) 026002 [hep-th/0102147] [INSPIRE].

[22] I. Olasagasti and K. Tamvakis, Gravity in higher codimension de Sitter brane worlds, Phys. Rev. D 68 (2003) 064016 [hep-th/0303096] [INSPIRE].

[23] C. Bachas and J. Estes, Spin-2 spectrum of defect theories, JHEP 06 (2011) 005 [arXiv: 1103.2800] [INSPIRE]. 
[24] L. Randall and R. Sundrum, An Alternative to compactification, Phys. Rev. Lett. 83 (1999) 4690 [hep-th/9906064] [INSPIRE].

[25] A. Karch and L. Randall, Locally localized gravity, JHEP 05 (2001) 008 [hep-th/0011156] [INSPIRE].

[26] W. Israel, Singular hypersurfaces and thin shells in general relativity, Nuovo Cim. B 44 (1966) 1 [Erratum ibid. 48 (1967) 463] [INSPIRE].

[27] C.P. Burgess, D. Hoover and G. Tasinato, UV Caps and Modulus Stabilization for $6 D$ Gauged Chiral Supergravity, JHEP 09 (2007) 124 [arXiv:0705.3212] [INSPIRE].

[28] C.P. Burgess, D. Hoover, C. de Rham and G. Tasinato, Effective Field Theories and Matching for Codimension-2 Branes, JHEP 03 (2009) 124 [arXiv:0812.3820] [INSPIRE].

[29] A. Bayntun, C.P. Burgess and L. van Nierop, Codimension-2 Brane-Bulk Matching: Examples from Six and Ten Dimensions, New J. Phys. 12 (2010) 075015 [arXiv:0912.3039] [INSPIRE].

[30] I.R. Klebanov and M.J. Strassler, Supergravity and a confining gauge theory: Duality cascades and chi SB resolution of naked singularities, JHEP 08 (2000) 052 [hep-th/0007191] [INSPIRE].

[31] I.R. Klebanov and A.A. Tseytlin, Gravity duals of supersymmetric $\mathrm{SU}(N) \times \mathrm{SU}(N+M)$ gauge theories, Nucl. Phys. B 578 (2000) 123 [hep-th/0002159] [INSPIRE].

[32] S. Weinberg, Gravitation and Cosmology, John Wiley and Sons, New York NY U.S.A. (1972).

[33] C.W. Misner, K.S. Thorne and J.A. Wheeler, Gravitation, W.H. Freeman, San Francisco CA U.S.A. (1973).

[34] C.P. Burgess and L. van Nierop, Large Dimensions and Small Curvatures from Supersymmetric Brane Back-reaction, JHEP 04 (2011) 078 [arXiv:1101.0152] [INSPIRE].

[35] I. Navarro and J. Santiago, Gravity on codimension 2 brane worlds, JHEP 02 (2005) 007 [hep-th/0411250] [INSPIRE].

[36] I.L. Buchbinder, D.M. Gitman, V.A. Krykhtin and V.D. Pershin, Equations of motion for massive spin-2 field coupled to gravity, Nucl. Phys. B 584 (2000) 615 [hep-th/9910188] [INSPIRE].

[37] S. Deser and A. Waldron, Stability of massive cosmological gravitons, Phys. Lett. B $\mathbf{5 0 8}$ (2001) 347 [hep-th/0103255] [INSPIRE].

[38] G.T. Horowitz and R.C. Myers, The AdS/CFT correspondence and a new positive energy conjecture for general relativity, Phys. Rev. D 59 (1998) 026005 [hep-th/9808079] [INSPIRE].

[39] J.M. Maldacena and C. Núñez, Supergravity description of field theories on curved manifolds and a no go theorem, Int. J. Mod. Phys. A 16 (2001) 822 [hep-th/0007018] [inSPIRE].

[40] I. Antoniadis, R. Minasian and P. Vanhove, Noncompact Calabi-Yau manifolds and localized gravity, Nucl. Phys. B 648 (2003) 69 [hep-th/0209030] [INSPIRE]. 